\title{
Multi-objective Optimization for Adaptive Web Site Generation
}

\author{
Prateek Jain $^{1}$ and Pabitra Mitra ${ }^{2}$ \\ 1 IBM India Research Lab, New Delhi - 110016, India \\ prateekj@in.ibm.com \\ 2 Department of Computer Science and Engineering, \\ Indian Institute of Technology, Kanpur -208016, India \\ pmitralitk.ac.in
}

\begin{abstract}
Designing web sites is a complex problem. Adaptive sites are those which improve themselves by learning from user access patterns. In this paper we have considered a problem of index page synthesis for an adaptive website and framed it in a new type of Multi-Objective Optimization problem. We give a solution to index page synthesis which uses a popular clustering algorithm DBSCAN alongwith NSGA-II-an evolutionary algorithm-to find out best index pages for a website. Our experiments shows that very good candidate index pages can be generated automatically, and that our technique outperforms various existing methods such as PageGather, K-Means and Hierarchical Agglomerative Clustering.
\end{abstract}

\section{Introduction}

In todays society websites have become the primary source of information, but a major problem is to design a website so that it is lucid and presents its information in an efficient manner. The major problem here is that different users at different time have different goals and when a designer designs a website he has little or no idea of how this information is going to be used. Thus most of the times when complex web sites are surfed, an user has to traverse a number of pages before he obtains the relevant information. Thus comes the need for adaptive websites, websites that automatically improve their organization and presentation by learning from visitors' access patterns. Major problems posed here are, (a) Can adaptive websites do non-trivial adaptations and still lead to improvement?, (b) Can they be fully automated? To solve these problems an approach was suggested by Etzioni et al.[1], where he defined a problem called index page synthesis and have studied this problem empirically. An index page is a page consisting of links to a set of pages that cover a particular topic at a site, thereby leading to efficient navigation of site.

In this work, we have tried to solve index page synthesis problem by casting it in a new type of Multi-Objective Optimization Problem and compared our results to other standard algorithms. Our approach is to analyze Web Site's access logs to find groups of pages that are coherent i.e. occur together in user's visits. If a group of pages generally occur together in a user's visit it means that they are similar and if we link them from each other then user will reach to information required easily. Thus a technique 
is required to cluster similar pages and then there should be a mechanism to evaluate weblogs to determine if the clustering is good or not, and this information can be used to further improve clustering. Unsupervised learning algorithms such as clustering algorithms are of much importance here. Also, after clustering there should be a fine tuning mechanism which improves these clusters. In our solution, we use the DBSCAN algorithm [2] initially for finding close clusters and then use NSGA-II algorithm[3] for optimizing these set of clusters. Generally Multi-Objective Optimization algorithms are used to optimize functions which are independent of each other. But here our problem have set of functions which depend on each other too. A new method has been devised to handle this situation. Results obtained by empirical studies show that our system outperforms the existing methods.

\section{Adaptive Wesites: Index-Page Synthesis}

A number of different of approaches exists in field of adaptive websites. Two popular among them are path prediction and index page synthesis approach. In path prediction, the website guesses the user intent from user profile and tries to direct user to that page. This is used in the Web Watcher and AVANTI systems [4]5]. The index page synthesis approach, introduced by Etzioni et. al.[1], will be used by us.

We can define Index-Page Synthesis problem as: given a Web site and a visitor access log, create new index pages containing collections of links to related but currently unlinked pages. By visitor access $\log$ we mean log generated by web-server, which contains one entry for each page requested of the web-server. Each entry contains visitor's IP-Address, URL requested, time of request. Related but currently unlinked pages are pages that share a common topic but are not currently linked at the site. Two pages are considered to be linked if there exists a link from one page to the other or if there exist a page that links to both them. We are stressing on unlinked pages, because if two pages are linked then user can anyhow reach from one to other very easily. Index Page Synthesis Problem can be decomposed into following major subproblems: (i) What are the contents(i.e hyper-link) of the index page?, (ii) How are the hyper-links labelled?, (iii) What is the title of the page and does it correspond to a coherent concept?, and (iv) Where index page should be added to the site? To solve first problem we do clustering of pages, so that pages which are of related content gets clustered together and thus they form content of one index page. Hyper-links are labelled by name of target page to which they link. Third and fourth problems are handled by webmaster.

Etzioni et al.[1] suggested following criterion for evaluation of effectiveness of index page synthesis: (a) Recall: How much of the information sought by each user was actually found, (b) Impact: How many people use the new pages and how often, (c) Benefit: How much effort is saved by those who visit the page.

In our study we have compared competing solutions with respect to impact and benefit. For each cluster, we count the number of pages in the cluster viewed by each visitor to the site, and compute the total number of visitors who view at least one page, the number who view at least two pages, and so on. We then average over all the clusters generated by a particular algorithm. For each particular algorithm, we plot a line of the number of pages viewed(benefit) vs. the number of people who viewed that many 
pages(impact). If no users viewed more than $m$ pages from any cluster for an algorithm, then the line for that algorithm will stop at $m$. We have represented impact on a log scale, as it often drops off exponentially with increasing benefit. Since index pages should not be too large or too small thus candidate index pages size have minimum and maximum size( 5 and 100 respectively).

\section{Proposed Approach for Index-Page Synthesis}

In order to generate index-page, first we need to preprocess web logs and calculate co-occurrence frequency, followed by clustering and optimization of the results.

\subsection{Pre-processing of Web Logs and Calculation of Co-occurrence Frequency}

An web log is a sequence of page views, or requests made to the Web server. Each request includes time of request, URL requested, and the machine from which the request originated. Assuming each originating machine corresponds to a single visitor, single session for that visitor is defined as series of page views in one day's log. Thus we define a visit as ordered sequences of pages accessed by a single user in a single session. Now, co-occurrence frequency[1] of two pages $p_{1}$ and $p_{2}$ is defined as $\min \left(P\left(p_{1} \mid p_{2}\right)\right.$, $\left.P\left(p_{2} \mid p_{1}\right)\right) . P\left(p_{1} \mid p_{2}\right)$ means probability of a visitor visiting $p_{1}$ if he/she has already visited $p_{2}$. We now form co-occurrence matrix, $C$, of size $n \times n$ ( $n$ is total number of pages in website), where $j$ th element of $i$ th row, $c_{i j}$, represents co-occurrence frequency of Page $p_{i}$ and Page $p_{j}$. Since we need to find out clusters of related but currently unlinked pages, thus to avoid finding cluster of pages already linked together, we set corresponding entries of linked pages as 0 .

\subsection{Clustering}

We use DBScan clustering algorithm [2] for clustering similar pages. DBScan algorithm is a density based clustering algorithm, whose main principle is that if some MinPts number of points are within $\epsilon$ distance of a given points then all the points belong to same cluster. Assuming pages which occur together are similar to each other, we can cluster them using DBScan algorithm, to obtain clusters of pages similar to each other. To do clustering, we have to first form feature space of each page. Each page is a point in $n$ dimensional space, where $i$ th co-ordinate corresponds to co-occurrence frequency of this page and $p_{i}$ page.

Performance of DBScan algorithm depends largely on two parameters i.e. $\epsilon$ and MinPts. These parameters should be set such that quality of clusters, i.e. Impact and benefit, produced is very good. This necessitates use of an optimizer which sets $\epsilon$ and MinPts, such that Impact and benefit are maximized.

\subsection{Multi-objective Optimization}

Since, the functions to be optimized are greater than one, it is a case of multi-objective optimization, with two objectives, namely Impact and benefit. But here there is a tradeoff. As benefit increases, impact decreases for a given $\epsilon$ and MinPts. So $f(\epsilon$, MinPts $)$ is a curve and not a point. Here $x$ co-ordinate represents benefit and $y$ co-ordinate represents impact. We have 


$$
\text { Benefit }\left(x_{i}\right)=i \text { for } 0 \leq i \leq m,
$$

where $m$ is maximum number of pages which at least one user visited. Hence, we need to apply optimizer so that curve is maximized. Now first we have to define what do we mean by maximization of a curve. Define,

$$
\begin{aligned}
\text { Curve } C_{1}(\epsilon, \text { MinPts }) & =\left\{\left(x_{1}, y_{1}\right),\left(x_{2}, y_{2}\right), \ldots,\left(x_{m}, y_{m}\right)\right\} \\
\text { Curve } C_{2}\left(\epsilon^{\prime},\right. \text { MinPts } & =\left\{\left(x_{1}^{\prime}, y_{1}^{\prime}\right),\left(x_{2}^{\prime}, y_{2}^{\prime}\right), \ldots,\left(x_{m^{\prime}}^{\prime}, y_{m}^{\prime}\right)\right\}
\end{aligned}
$$

If $m^{\prime}<m$, then extend Curve $C_{2}$ to $x_{m}^{\prime}$ with $y_{j}^{\prime}=0$ for $m^{\prime}<j \leq m$. Now, we define a partial ordering on curves, $C_{1} \geq C_{2}$ iff $\forall i \leq m \quad y_{i} \geq y_{i}^{\prime}$, else no relation is defined between $C_{1}$ and $C_{2}$.

We use a multiobjective algorithm named Non-Dominated Sorting Genetic Algorithm (NSGA-II) for optimization, which is an population based iterative algorithm . NSGA-II is based on principle of non-dominance of points i.e. let there are two variables $x$ and $y$, and we have to maximize $f_{1}(x, y)$ and $f_{2}(x, y)$. Points $\left(x_{1}, y_{1}\right)$ is dominated by $\left(x_{2}, y_{2}\right)$ iff $f_{1}\left(x_{1}, y_{1}\right) \geq f_{1}\left(x_{2}, y_{2}\right)$ and also $f_{2}\left(x_{1}, y_{1}\right) \geq f_{2}\left(x_{2}, y_{2}\right)$. NSGA-II proceeds with a random generation of solutions $(x, y)$ called population. Then a selection is applied to the generated solutions which takes out the best solutions out of current population. Best solutions are those which are least dominated. So from these best solutions, new solutions are formed using crossover operator and mutation operator and then this forms new population. Thus this process of selection and reproduction is continued till some terminating condition is satisfied.

For our problem, we have two variables namely $\epsilon$ and MinPts, which determines the clusters obtained. Quality of these clusters give curve of Impact vs Benefit. To apply NSGA-II in order to find out set of best $\epsilon$ and MinPts, we have to define domination of $(\epsilon$, MinPts $) .(\epsilon$, MinPts $)$ dominates $\left(\epsilon^{\prime}\right.$, MinPts $\left.s^{\prime}\right)$ iff $C_{1}(\epsilon$, MinPts $) \geq$ $C_{2}\left(\epsilon^{\prime}\right.$, MinPts $\left.s^{\prime}\right)$ else $\left(\epsilon^{\prime}\right.$, MinPts $\left.s^{\prime}\right)$ and $(\epsilon$, MinPts $)$ are non-dominated by each other, $C_{1}(\epsilon, M i n P t s)$ is produced by finding out cluster's(which is produced by applying DBScan with parameters $\epsilon$ and MinPts) impact vs benefit curve. Now we can directly apply NSGA-II to get optimized set of solutions.

Note that no curve can be said better than other in Pareto optimal set according to given objective functions. But after looking at all the pareto optimal curves, webmaster can decide to pick up one solution depending on targets of websites. For example a website which is targeted for a large number of visitors they will prefer curve which have high impact for benefit of 1 or 2 pages, though for higher benefits impact is not much. E.g. a website selling cheap electronic goods, not having alot of choices in products, will like that a large number of customers are attracted towards it though it maybe that they don't buy a lot of products. Where as websites for luxury cars will like to entice a few customers, but make sure that all of them get to know all the different varieties and are completely satisfied by their purchases.

\section{Experimental Results and Comparison}

We test our system on data collected from webpages of website Music Machine. Music Machine is a site devoted to information about many kinds of electronic musical 
instruments. Music Machines contains approximately 2500 distinct pages, including HTML pages, plain text, images, and audio samples. Music Machines receives approximately 10,000 hits per day from roughly 1200 distinct visitors. In our experiments, the training data is a collection of access logs for six months; the test data is a set of logs from a subsequent one-month period. NSGA-II is run for 50 Generation and 50 Population.

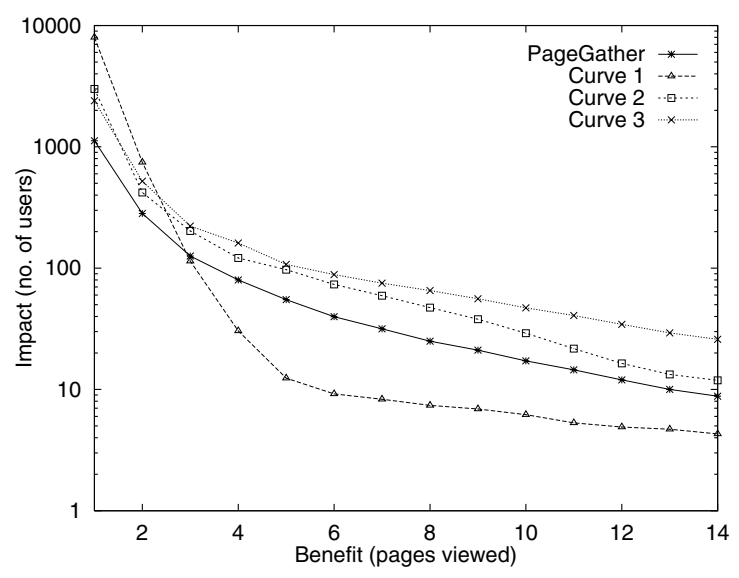

Fig. 1. Comparison of our algorithm and PageGather Algorithm

We compare our results with that of PageGather Algorithm which performed better than standard algorithms like K-Means, Hierarchial Agglomerative Clustering. Figure1 shows Impact vs Benefit curve for both our system and for PageGather. We have compared our algorithm with PageGather Algorithm since it is known to perform better than the other algorithms [1].

Note that our algorithm gives a set of curves rather than just a single curve. These curves gives a wide range of choice to Webmaster to choose the one which suits his website the most. In the graph we have reported just few of those solutions else it would have become grossly confusing. Observe that out of set of pareto- optimal curves, two curves outperforms PageGather algorithm completely. Also none of our pareto-optimal curves were dominated by PageGather algorithm's solution.

\section{Conclusion}

The index page synthesis problem for adaptive web site design is formulated as a multiobjective optimization problem. A modified MOGA is used to optimize a page clustering clustering algorithm based on DBScan so as to give near optimal results on two parameters namely Impact and Benefit. Our better results goes onto indicate that Evolutionary Algorithms are quite suited to Webmining and Weblearning problems and can be used to obtain much improved performance than the present techniques. 


\section{References}

1. Perkowitz, M., Etzioni, O.: Towards adaptive web sites: Conceptual framework and case study. Computer Networks 31 (1999) 1245-1258

2. Ester, M., Kriegel, H.P., Sander, J., Xu, X.: A density-based algorithm for discovering clusters in large spatial databases with noise. In: KDD. (1996) 226-231

3. Schoenauer, M., Deb, K., Rudolph, G., Yao, X., Lutton, E., Guervós, J.J.M., Schwefel, H.P., eds.: Parallel Problem Solving from Nature - PPSN VI, 6th International Conference, Paris, France, September 18-20, 2000, Proceedings. In Schoenauer, M., Deb, K., Rudolph, G., Yao, X., Lutton, E., Guervós, J.J.M., Schwefel, H.P., eds.: PPSN. Volume 1917 of Lecture Notes in Computer Science., Springer (2000)

4. Joachims, T., Freitag, D., Mitchell, T.M.: Web watcher: A tour guide for the world wide web. In: IJCAI (1). (1997) 770-777

5. Fink, J., Koenemann, J., Noller, S., Schwab, I.: Putting personalization into practice. Commun. ACM 45 (2002) 41-42 